\title{
IM2GPS: estimating geographic information from a single image
}

\author{
James Hays and Alexei A. Efros \\ Carnegie Mellon University
}

\begin{abstract}
Estimating geographic information from an image is an excellent, difficult high-level computer vision problem whose time has come. The emergence of vast amounts of geographically-calibrated image data is a great reason for computer vision to start looking globally - on the scale of the entire planet! In this paper, we propose a simple algorithm for estimating a distribution over geographic locations from a single image using a purely data-driven scene matching approach. For this task, we will leverage a dataset of over 6 million GPS-tagged images from the Internet. We represent the estimated image location as a probability distribution over the Earth's surface. We quantitatively evaluate our approach in several geolocation tasks and demonstrate encouraging performance (up to 30 times better than chance). We show that geolocation estimates can provide the basis for numerous other image understanding tasks such as population density estimation, land cover estimation or urban/rural classification.
\end{abstract}

\section{Introduction}

Consider the photographs in Figure 1. What can you say about where they were taken? The first one is easy - it's an iconic image of the Notre Dame cathedral in Paris. The middle photo looks vaguely Mediterranean, perhaps a small town in Italy, or France, or Spain. The rightmost photograph is the most ambiguous. Probably all that could be said is that it's a picture of a seaside in some tropical location. But note that even this vague description allows us to disregard all non-coastal, non-tropical areas - more than 99.9\% of the Earth's surface! Evidently, we humans have learned a reasonably strong model for inferring location distribution from photographs. Moreover, even in cases when our geo-localization performance is poor, we are still able to give fairly confident estimates to other related questions: How hot/cold does it get? How many people live there? How well-off are they? etc.

What explains this impressive human ability? Semantic reasoning, for one, is likely to play a big role. People's faces and clothes, the language of the street signs, the types of trees and plants, the topographical features of the terrain - all can serve as semantic clues to the geographic location of a particular shot. Yet, there is mounting evidence in cognitive science that data association (ask not "What is it?" but rather "What is it like?") may play a significant role as well [1]. In the example above, this would mean that instead of reasoning about a beach scene in terms of the trop-

\footnotetext{
${ }^{1}$ Project Page: http://graphics.cs.cmu.edu/projects/im2gps/
}

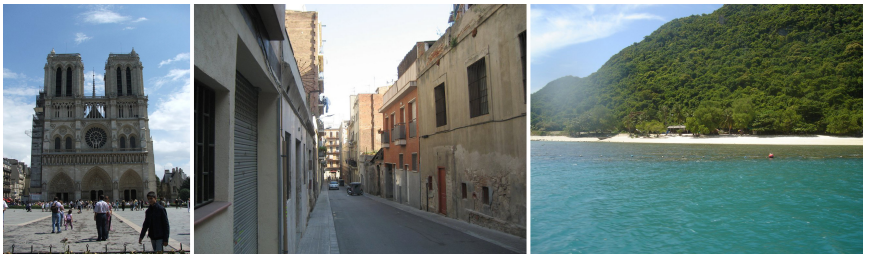

Figure 1. What can you say about where these photos were taken?

ical sea, sand and palm trees, we would simply remember: "I have seen something similar on a trip to Hawaii!". Note that although the original picture is unlikely to actually be from Hawaii, this association is still extremely valuable in helping to implicitly define the type of place that the photo belongs to.

Of course, computationally we are quite far from being able to semantically reason about a photograph (although encouraging progress is being made). On the other hand, the recent availability of truly gigantic image collections has made data association, such as brute-force scene matching, quite feasible [17, 4].

In this paper, we propose an algorithm for estimating a distribution over geographic locations from an image using a purely data-driven scene matching approach. For this task, we leverage a dataset of over 6 million GPS-tagged images from the Flickr online photo collection. We represent the estimated image location as a probability distribution over the Earth's surface, and geolocation performance is analyzed in several tasks. Additionally, the usefulness of image localization is demonstrated with meta-tasks such as land cover estimation and urban/rural classification.

\subsection{Background}

Visual localization on a topographical map has been one of the early problems in computer vision, which turned out to be extremely challenging for both computers and humans [16]. But the situation improves dramatically if more sources of data are available. Jacobs et al. [6] proposes a very clever and simple method of geolocating a webcam based on correlating its video-stream with satellite weather maps over the same time period.

The recent availability of GPS-tagged images of urban environments coupled with advances in multi-view geometry and efficient feature matching led to a number of groups developing place recognition algorithms, some of which competed in the "Where am I?" Contest [15] at ICCV'05 (winning entry described in [19]). Similar feature-based geometric matching approaches have also been successfully applied to co-registering online photographs of famous landmarks for browsing [14] and summarization [13], 

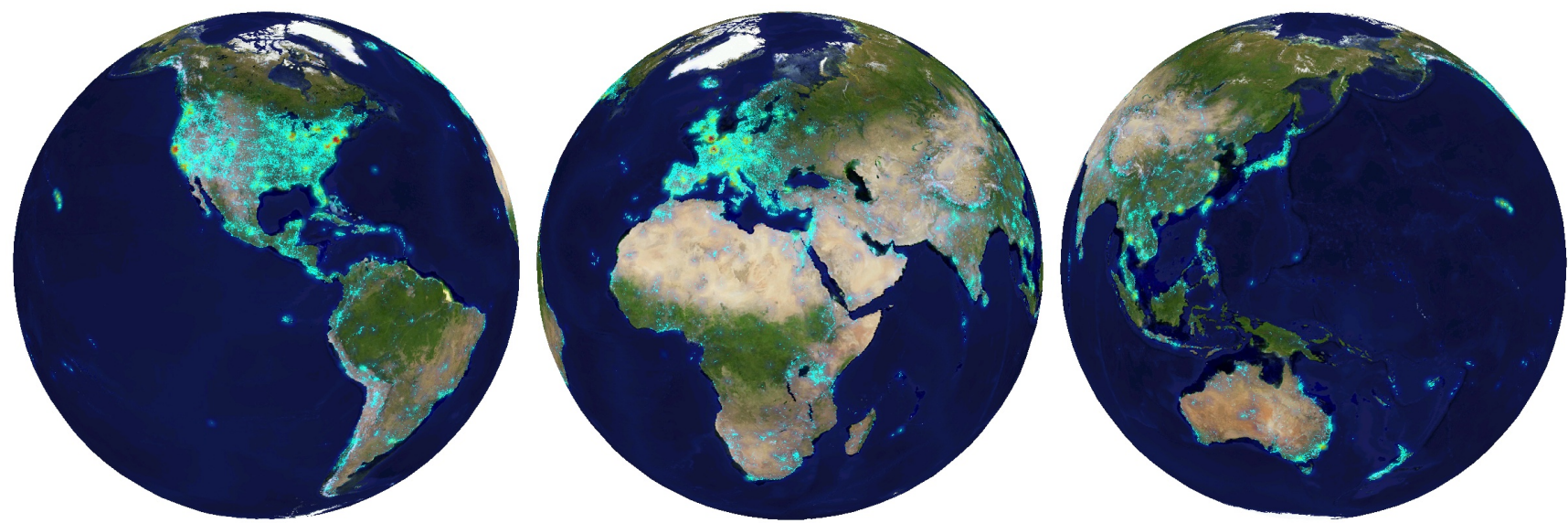

Figure 2. The distribution of photos in our database. Photo locations are cyan. Density is overlaid with the jet colormap (log scale).

as well as image retrieval in location-labeled collections, e.g. [2].

But can these geometric feature-based matching approaches scale up to the entire world? This is unlikely, not just because of computational cost, but simply because the set of all existing photographs is still not large enough to exhaustively sample the entire world. Yes, there would be tens of thousands of photos of a famous landmark, but some ordinary streets or even whole cities might be entirely missing. And since the geometric constraints require an exact match, most of the time the system will retrieve nothing at all. Clearly, a generalization of some sort is required.

On the other side of the spectrum is the philosophy that all forests look more or less the same, as do deserts, mountains, cities, kitchens, bathrooms, etc. A large body of work exist on scene recognition $[10,12,8,18]$, which involves defining a handful of scene categories and using various low-level features to classify a novel image into one of these categories. While impressive results are typically obtained, classification is not a difficult task if the number of categories is small. Moreover, the choice of categories is often not very scientific.

The approach we are proposing in this paper neatly straddles these two extremes, seamlessly adapting to the amount of data available. If the query image is a famous landmark, there will likely be many similar images of the same exact place in the database, and our approach is likely to return a precise GPS location. If the query is more generic, like a desert scene, many different deserts will match, producing a location probability that is high over the dry, sandy parts of the world. In fact, our approach provides a more scientifically valid method of defining scene categories - based on geographic location as well as appearance.

\section{Building a Geo-tagged Image Dataset}

In order to reason about the global location of an arbitrary scene we first need a large number of images that are labelled with geographic information. This information could be in the form of text keywords or it could be in the form of GPS coordinates. Fortunately there is a huge (and rapidly growing) amount of online images with both types of labels. For instance, Flickr.com has hundreds of millions of pictures with either geographic text or GPS coordinates.

But it is still difficult to create a useful, high-quality database based on user collected and labelled content. We are interested in collecting images that depict some amount of geographic uniqueness. For instance, pictures taken by tourists are ideal because they often focus on the unique and interesting qualities of a place. Many of these images can be found because they often have geographic keywords associated with them (i.e. city or country names). But using geographic text labels is problematic because many of them are ambiguous (e.g. Washington city/state, Georgia state/country, Mississippi river/state, and LA city/state) or spatially broad (e.g. Asia or Canada).

Images annotated with GPS coordinates are geographically unambiguous and accurate, but are more likely to be visually irrelevant. Users tend to geo-tag all of their pictures, whether they are pet dog pictures (less useful) or hiking photos (more useful). In fact, the vast majority of online images tagged with GPS coordinates and to a lesser extent those with geographic text labels are not useful for image-based geolocation. Many of the images are poor quality (low resolution, noisy, black and white) or depict scenes which are only marginally useful for geolocation (most portraits, wedding pictures, abstracts, and macro photography). While these types of photos can sometimes reveal geographic information (western-style weddings are popular in Europe and Japan but not India; pet dogs are popular in the US but not Syria) the customs are so broadly distributed that it is not very useful for geolocation.

However, we found that by taking the intersection of these groups, images with both GPS coordinates and geographic keywords, we greatly increased the likelihood of finding accurately geolocated and visually useful data. People may geo-tag images of their cats, but they're less likely to label that image with "New York City" at the same time. 


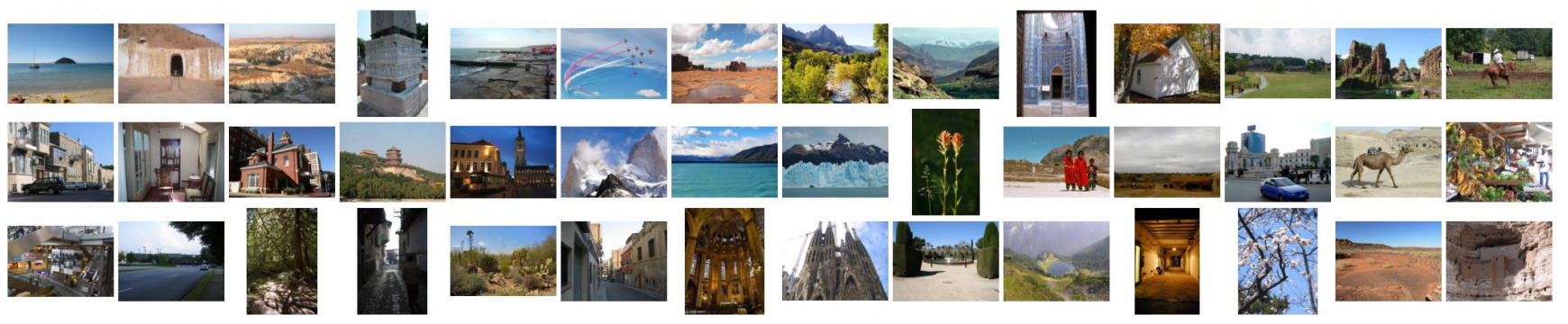

Figure $3.18 \%$ of our 237 image test set. Note how difficult it is to specifically geolocate most of the images.

Our list of geographic keywords includes every country and territory, every continent, the top 200 most populated cities in the world, every US state, and popular tourist sites (e.g. "Pisa", "Nikko", "Orlando").

This results in a pool of approximately 20 million geotagged and geographic text-labelled images from which we excluded all photos which were also tagged with keywords such as "birthday", "concert", "abstract" and "cameraphone". In the end we arrived at a database of $6,472,304$ images. All images were downsized to max dimension 1024 and JPEG compressed for a total of 1 terabyte of data.

While this is a tremendous amount of data it cannot be considered an exhaustive visual sampling of Earth. Our database averages only 0.0435 pictures per square kilometer of Earth's land area. But as figure 2 shows the data is very non-uniformly distributed towards places where people live or travel which is fortunate since geolocation query images are likely to come from the same places.

\subsection{Evaluation Test Set}

To evaluate the performance of our method, we also need a separate hold-out test set of geo-located images. We built the test set by drawing 400 random images from the original data set. From this set we manually removed any undesirable photos that were not automatically excluded during database construction - abstract photos, overly processed or artistic photos, and black and white photos. We also excluded photos with significant artifacts such as motion blur or extreme noise. Finally we removed pictures with easily recognizable people or other situations that might violate someone's privacy. To ensure that our test set and database are independent we exclude from the database not just test images, but all other images from the same photographers.

Of the 237 resulting images, about $5 \%$ are recognizable as specific tourist sites around the globe but the great majority are only recognizable in a generic sense (Figure 3 shows a random sample of test set). Some of the images contain very little geographic information, even for an astute human examiner. We think this test set is extremely challenging but representative of the types of photos people take.

\section{Scene Matching}

Is it feasible to extract geographic information from generic scenes? One of the main questions addressed by this paper is as much about the Earth itself as it is about com- puter vision. Humans and computers can recognize specific, physical scenes that they've seen before, but what about more generic scenes which make up most of our database and our test set. Many of these scenes may be impossible to specifically localize. We know that our world is self-similar not just locally but across the globe. Film creators have long taken advantage of this (e.g. "Spaghetti Westerns" films that were ostensibly set in the American Southwest but filmed in Almería, Spain.) Nonetheless, it must be the case that certain visual features in imagery correlate strongly with geography even if the relationship is not strong enough to specifically pinpoint a location. Beach images must be near bodies of water, jungles must be near the equator, and glaciated mountains cover a relatively small fraction of the Earth's surface.

What features can we extract from images that will best allow us to examine and exploit this correlation between image properties and geographic location? In this paper we evaluate an assortment of popular features from literature:

Tiny Images: The most trivial way to match scenes is to compare them directly in color image space. Reducing the image dimensions drastically makes this approach more computationally feasible and less sensitive to exact alignment. This method of image matching has been examined thoroughly by Torralba et al.[17] for the purpose of object recognition and scene classification. Inspired by this work we will use 16 by 16 color images as one of our features.

Color histograms: In the spirit of most image retrieval literature, we build joint histograms of color in CIE L*a*b* color space for each image. Our histograms have 4, 14, and 14 bins in L, a, and b respectively for a total of 784 dimensions. We have fewer bins in the intensity dimension because other descriptors will measure the intensity distribution of each image. We compute distance between these histograms using $\chi^{2}$ distance.

Texton Histograms: Texture features might help distinguish between geographically correlated properties such ornamentation styles or building materials in cities or vegetation and terrain types in landscapes. We build a 512 entry universal texton dictionary [9] by clustering our dataset's responses to a bank of filters with 8 orientations, 2 scales, and 2 elongations. For each image we then build a 512 dimensional histogram by assigning each pixel's set of filter responses to the nearest texton dictionary entry. Again, we 
use $\chi^{2}$ distances between texton histograms. Note that this representation is quite similar to dense visual words.

Line Features: We have found that the statistics of straight lines in images are useful for distinguishing between natural and man-made scenes and for finding scenes with similar vanishing points. We find straight lines from Canny edges using the method described in Video Compass [7]. For each image we build two histograms based on the statistics of detected lines- one with bins corresponding to line angles and one with bins corresponding to line lengths. We use L1 distance to compare these histograms.

Gist Descriptor + Color: The gist descriptor [11] has been shown to work well for scene categorization [10] and for retrieving semantically and structurally similar scenes [4]. We create a gist descriptor for each image with 5 by 5 spatial resolution where each bin contains that image region's average response to steerable filters at 6 orientations and 4 scales. We also create a tiny $\mathrm{L} * \mathrm{a} * \mathrm{~b}$ image, also at 5 by 5 spatial resolution.

Geometric Context: Finally, we compute the geometric class probabilities for image regions using the method of Hoiem et al. [5]. We use only the primary classes- ground, sky, and vertical since they are more reliably classified. We reduce the probability maps for each class to $8 \times 8$ and use L2 distance to compare them.

We precomputed all features for the database which took about 15 seconds per image on a contemporary Xeon processor for a total of $3.08 \mathrm{CPU}$ years. Using a cluster of 400 processors we computed the features over 3 days.

\section{Data-driven Geolocation}

After all the preprocessing is complete, the geolocation framework is quite simple. For each input image in our test set we build the same features as discussed in Section 3 and compute the distance in each feature space to all 6 million images in the database. We scale each feature's distances so that their standard deviations are roughly the same and thus they influence the ordering of scene matches equally. For each query image we use the aggregate feature distances to find the nearest neighbors in the database and we derive geolocation estimates from those GPS tagged nearest neighbors.

The simplest heuristic is to use the GPS coordinate of the first nearest neighbor (1-NN) as our geolocation estimate. Of course, 1-NN approaches are often not robust. Alternatively, we can consider a larger set of $k$-NN ( $k=120$ in our experiments). This set of nearest neighbors together forms an implicit estimate of geographic location - a probability map over the entire globe. The hope is that the location of peak density in this probability map corresponds to the true location of the query image. One way to operationalize this is to consider the major modes of the distribution by performing mean-shift [3] clustering on the geolocations of the matches. We represent the geolocations as $3 \mathrm{~d}$ points and reproject the mean-shift clusters to the Earth's surface after

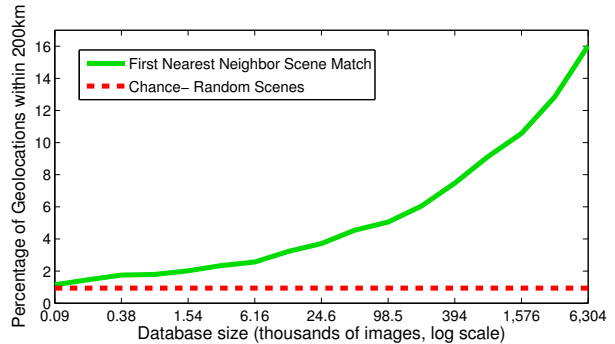

Figure 4. Geolocation performance across database sizes. Percentage of test set images that were correctly geolocated within $200 \mathrm{~km}$ of ground truth as function of dataset size using 1-NN. As the database shrinks the performance converges to chance.

the clustering procedure. We use a mean-shift bandwidth of $500 \mathrm{~km}$ (although other settings work similarly) and disregard clusters with fewer than 4 matches, resulting in between 6 to 12 clusters containing, on average, about two thirds of the original 120 matches. This serves as a kind of geographic outlier rejection to clean up spurious matches, but can be unfavorable to locations with few data-points.

To compute a geolocation estimate, one approach is to pick the cluster with the highest cardinality and report the GPS coordinate of its mode. For some applications, it might be acceptable to return a list of possible location estimates, in which case the modes of the clusters can be reported in order of decreasing cardinality. We show qualitative results for several images in Figure 15. More results can be found on our project web page.

\subsection{Is the data helping?}

The most interesting research question for us is how strongly does image similarity correlate with geographic proximity? To geolocate a query we don't just want to find images that are similarly structured or of the same semantic class (e.g. "forest" or "indoors"). We want image matches that are specific enough to be geographically distinct from otherwise similar scenes. How much data is needed start to capture this geography-specific information? In Figure 4 we plot how frequently the 1-NN scene match is within $200 \mathrm{~km}$ of the query's true location as we alter the size of the database. With a tiny database of 90 images, the 1-NN scene match is as likely to be near the query as a random image from the database. With the full database we perform 18 times better than chance. Note that the percentage of test cases geolocated to within $200 \mathrm{~km}$ (16\%) is significantly higher than the proportion of "landmark" images (e.g. Notre Dame) in the test set (about 5\%).

\subsection{Which features are most geo-informed?}

Another interesting question we consider is which visual characteristics are more helpful in disambiguating between locations? In Figure 5 we examine the geolocation accuracy when using each of the features from Section 3 in isolation as well as in unison. For each feature we consider the geolocation accuracy of 1-NN against the largest cluster. The latter is indeed more robust than using 1-NN, although per- 


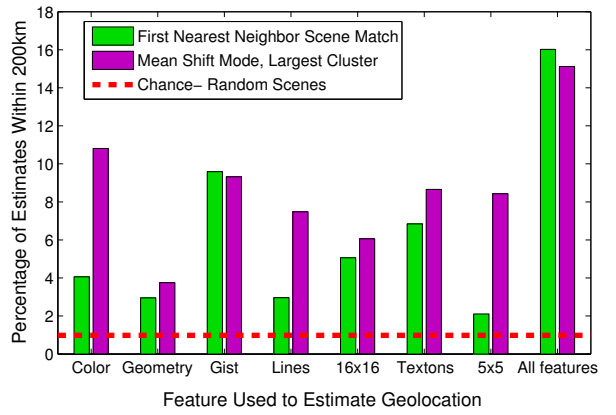

Figure 5. Geolocation performance across features. Percentage of test cases geolocated to within $200 \mathrm{~km}$ for each feature. We compare geolocation by $1-\mathrm{NN}$ vs. largest mean-shift mode.

formance is similar when using all features. The richer feature combinations seem less prone to geographic outliers which disrupt the 1-NN approach.

Using all features together performed considerably better than any one by itself, suggesting that the information they are capturing is somewhat independent. The most geographically discriminative features are the gist, color histogram, and texton histogram. The gist, especially, performs well, even in the $1-\mathrm{NN}$ regime, reaffirming its position as the feature of choice for scene matching tasks. Surprisingly, color also does extremely well (but only after discarding geographic outliers), which suggests that it is a more diverse and location-specific feature than previously assumed (artists have long talked about "that special color" of a particular location). The least geographically discriminative feature is the $8 \times 8$ geometric context class likelihoods. This seems reasonable - the geometric context framework is inspired by the observation that the vast majority of scenes can be succinctly modelled by ground, sky, and vertical components. A view down a forest path can share the same class distribution as a view down Wall St. (when considering only the primary classes). The 16x16 tiny images also scored low. In fact, after geographic outlier rejection, they performed worse than humble $5 \times 5$ color images, suggesting that perhaps they are too noisy for this task. In the rest of the evaluations, we will use all features except the geometric context and the 16x16 tiny images.

\subsection{How accurate are the estimates?}

Given a photo, how often can we pin-point the right city? Country? Continent? So far we have evaluated geolocation accuracy only in terms of a distance threshold. In Figure 6 we more closely examine the distribution of geolocation errors (distances between estimated and ground truth locations) across our test set. For the two heuristics (1-NN, and mean-shift mode) plus three baselines (chance, and two best case scenarios), we sort the errors on the test set independently, from best to worst. We see that both heuristics are able to localize about $25 \%$ of the data within the scale of a (small) country. While 1-NN approach performs better at precise localization (within a city), mean-shift mode does

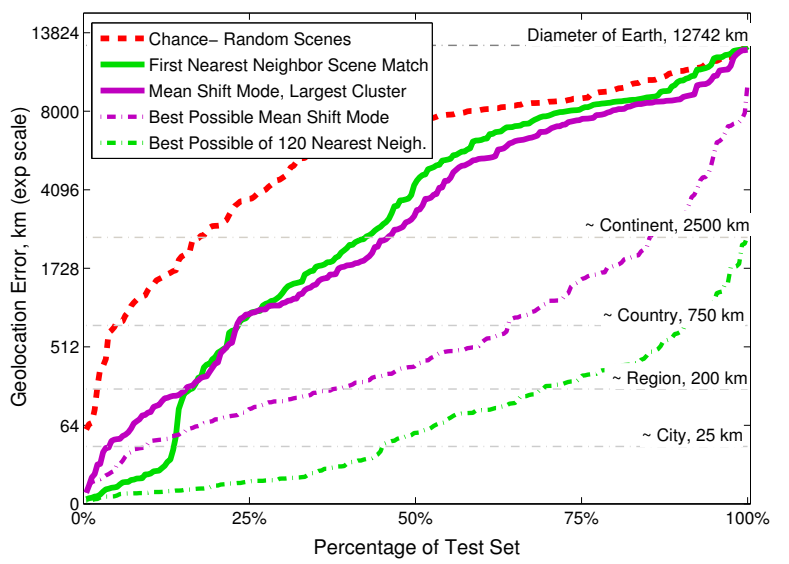

Figure 6. Accuracy of geolocation estimates across the test set. Localization errors (distance between predicted and ground truth location), are shown for 1-NN and mean-shift estimates. Errors are sorted from best to worst independently for each curve, thus showing the proportion of images geolocated within any error threshold. Chance performance (random matches) and two best-case scenarios - picking the mean-shift mode or scene match which is spatially closest to the ground truth query location - are shown for comparison.

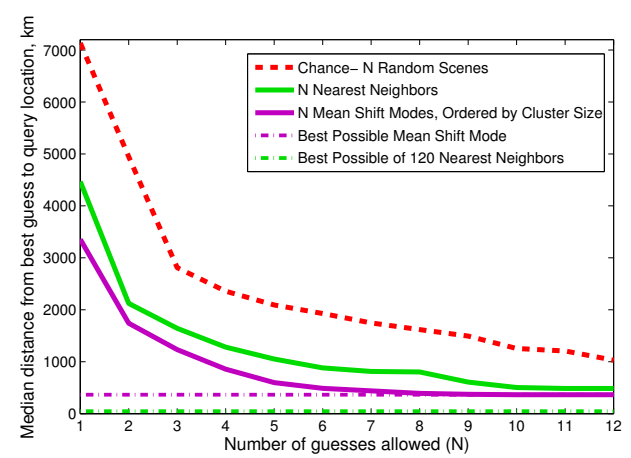

Figure 7. Geolocation error with multiple guesses. Median geolocation error for NN and mean-shift modes with increasing numbers of guesses allowed. The error is the distance from an algorithm's best guess to the query's ground truth location. Although the geolocation of a query may be ambiguous among several possibilities, after multiple guesses it is likely that one of the estimates is near the ground truth location.

somewhat better for more global localization. Both methods outperform chance by a large margin for two thirds of the test cases.

It's instructive to note that although the largest one-third of errors across the test set are very large (nearly as bad a chance), for almost all queries there is some scene match or mean-shift cluster that is quite close to the query (see "best case" curves on Figure 6). In other words, among the 120 nearest neighbors there are almost always several geographically accurate matches but the heuristics sometimes have trouble disambiguating those from other visually similar, spatially dissimilar matches (e.g. Hawaii vs Martinique or New York City vs Hong Kong). If we allow ourselves $N$ 


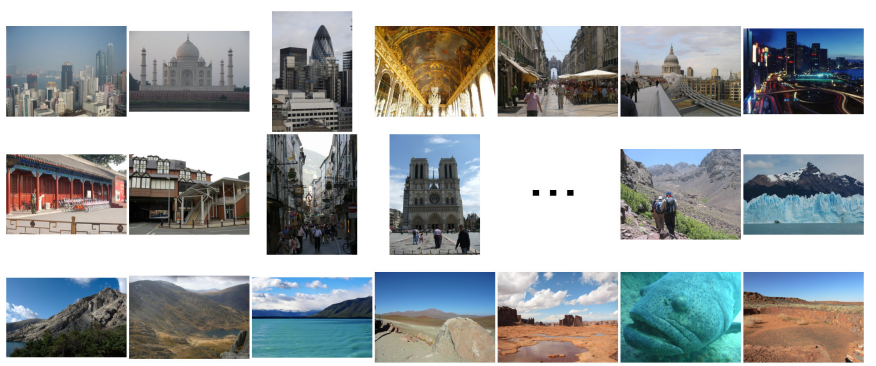

Figure 8. In scanline order, the test cases with the highest and lowest estimated population density.
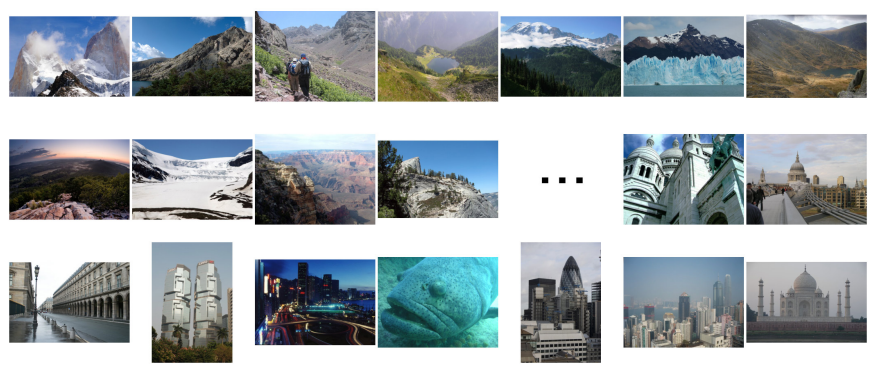

Figure 9. In scanline order, the test cases with the largest and smallest estimated elevation gradient.

"guesses" as to the location of a query we can rapidly get closer to the ground truth location (Figure 7). For this task we compare geolocation estimates from $N$ nearest neighbors and the modes of the $N$ largest clusters. The probability map modes are especially accurate for this task. With 6 guesses for each query, the median error for the test set is less than $500 \mathrm{~km}$ (nearly half the error of $6-\mathrm{NN}$, and one quarter the error of 6 random scenes).

\section{Secondary Geographic Tasks}

Once we have a geolocation estimate (either in the form of a specific location estimate or a probability map), we can use it to index into any geographic information system (GIS). There is a vast amount of freely available geolocated information about our planet such as climate information, crime rates, real estate prices, carbon emissions, political preference, etc. Even if an image cannot be geolocated accurately, its geographic probability map might correlate strongly with some features of the planet. For instance, given a map of population density (P.D.) and a query image, we can sample the P.D. map at the estimated geolocation(s) and use the average value as a P.D. estimate for the query image. Using this approach we estimate the population density (Figure 8) and elevation gradient magnitude (Figure 9) for each of our 237 test images.

We also produce land cover estimates for each of our test images by sampling from a land cover classification map (Figure 10) according to each image's geolocation probability map. We show the test images which are most likely to be "forest" (Figure 11), "water" (Figure 12), and "savanna" (Figure 13).

This framework can also be used to retrieve geograph-

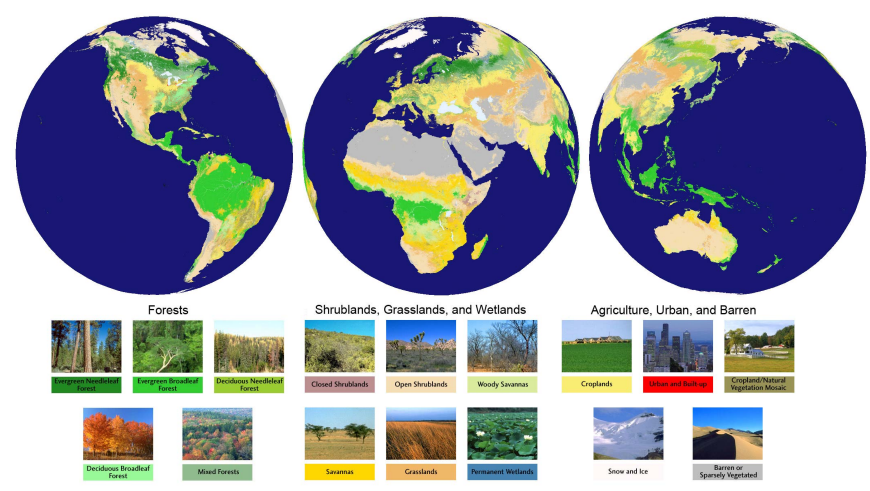

Figure 10. Land cover classification map and key.

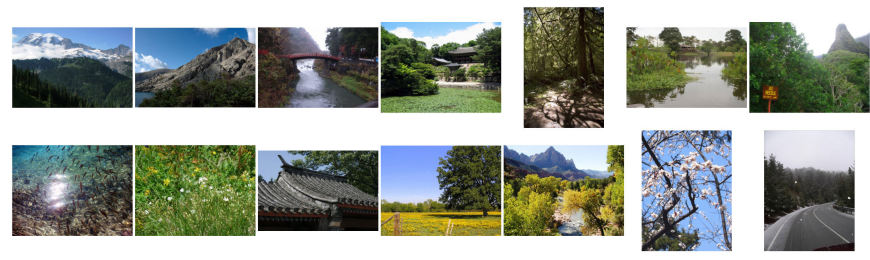

Figure 11. Test images with highest "forest" likelihood. Note that there is no "mountain" class in the land cover map- most mountains are labelled as "forest" or "barren" according to their land cover. The mountains above are indeed forested.

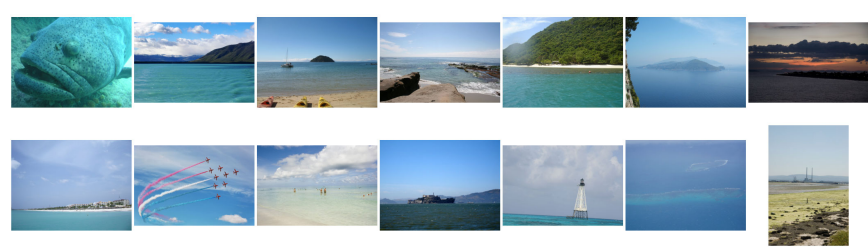

Figure 12. Test images with the highest "water" likelihood.

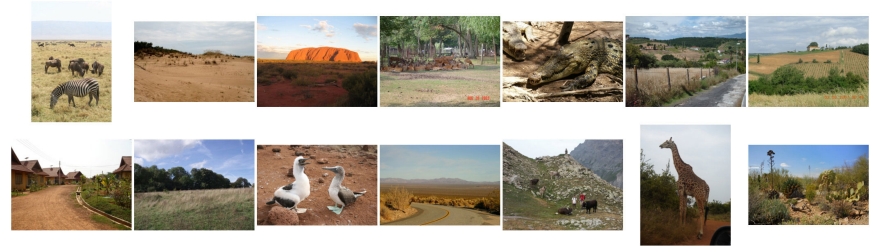

Figure 13. Test images with the highest "savanna" likelihood. Perhaps only a couple of the images in our test set actually depict "savanna", but many of these images contain similar geographic elements.

ically relevant images out of an unlabelled collection, i.e. "Which images in my photo collection are from my trip to India?". In this case the secondary geographic data source could be a global map where India $=1$.

Additionally, we can perform image classification using properties derived from a secondary geographical data source according to our geolocation estimates. For example, using a global map of light pollution (not shown), we look up the light pollution magnitude at the ground truth location of each of our test cases. We divide the test images along their median light pollution value into "urban" and "rural" classes. This is a difficult classification problem because the classes are not cleanly separated, but it is a more principled way to generate labelled data than has been 


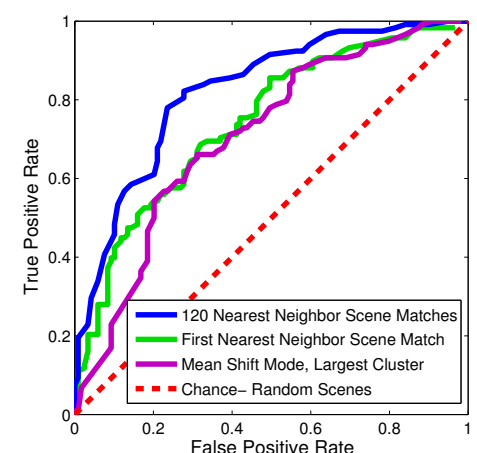

Figure 14. ROC curve for urban/rural classification. Areas under ROC curve are $.82, .74$, and .71 for $120-\mathrm{NN}, 1-\mathrm{NN}$, and 1 meanshift mode, respectively.

done in previous scene classification work. Having defined a ground truth classification, we try to predict each test image's class without using its ground truth location but instead using its estimated geolocation. Figure 14 shows the ROC curve for this task using different heuristics to estimate geolocation. Using the entire geolocation probability map instead of a single, explicit geolocation estimate performs best (.82 area under ROC).

\section{Discussion}

We believe that estimating geographic information from images is an excellent, difficult, but very much doable high-level computer vision problem whose time has come. The emergence of so much geographically-calibrated image data is an excellent reason for computer vision to start looking globally - on the scale of the entire planet! Not only is geo-location an important problem in itself, but it could also be tremendously useful to many other vision tasks:

i) Knowing the distribution of likely geolocations for an image provides huge amounts of additional meta-data for climate, average temperature for any day, vegetation index, elevation, population density, per capita income, average rainfall, etc.

ii) Even a coarse geo-location can provide a useful object prior for recognition. For instance, knowing that a picture is somewhere in Japan would allow one to prime object detection for the appropriate type of taxi cabs, lane markings, average pedestrian height, etc.

iii) Geo-location provides a concrete task that can be used to quantitatively evaluate scene matching algorithms as well as provide a more scientific basis for scene recognition studies, both for humans and machines.

In conclusion, this paper is the first to be able to extract geographic information from a single image. It is also the first time that a truly gargantuan database of over 6 million geolocated images has been used in computer vision. While our results look quite promising, much work remains to be done. We hope that this work might jump-start a new direction of research in geographical computer vision.

Acknowledgements: We thank Steve Schlosser, Julio Lopez, and Intel Research Pittsburgh for helping us overcome the logistical and computational challenges of this project. We thank Yahoo! and Flickr for sharing their computing and image resources with the research community. All visualizations and geographic data sources are derived from NASA data. Funding was provided by an NSF fellowship to James Hays and NSF grants CAREER IIS0546547 and CCF-0541230.

\section{References}

[1] M. Bar. The proactive brain: using analogies and associations to generate predictions. Trends in Cognitive Sciences, 11(7), 2007.

[2] O. Chum, J. Philbin, J. Sivic, M. Isard, and A. Zisserman. Total recall: Automatic query expansion with a generative feature model for object retrieval. In Proc. ICCV, 2007.

[3] D. Comaniciu and P. Meer. Mean shift: A robust approach toward feature space analysis. IEEE Trans. Pattern Anal. Mach. Intell., 24(5):603-619, 2002.

[4] J. Hays and A. A. Efros. Scene completion using millions of photographs. ACM Transactions on Graphics (SIGGRAPH 2007), 26(3), 2007.

[5] D. Hoiem, A. A. Efros, and M. Hebert. Geometric context from a single image. In Proc. ICCV, October 2005.

[6] N. Jacobs, S. Satkin, N. Roman, R. Speyer, and R. Pless. Geolocating static cameras. In Proc. ICCV, 2007.

[7] J. Kosecka and W. Zhang. Video compass. In ECCV '02: Proceedings of the 7th European Conference on Computer Vision-Part IV, pages 476-490, 2002.

[8] L.-J. Li and L. F. Fei. What, where and who? classifying events by scene and object recognition. In Proc. ICCV, 2007.

[9] D. Martin, C. Fowlkes, D. Tal, and J. Malik. A database of human segmented natural images and its application to evaluating segmentation algorithms and measuring ecological statistics. In Proc. ICCV, July 2001.

[10] A. Oliva and A. Torralba. Modeling the shape of the scene: A holistic representation of the spatial envelope. Int. J. Comput. Vision, 42(3):145-175, 2001

[11] A. Oliva and A. Torralba. Building the gist of a scene: The role of global image features in recognition. In Visual Perception, Progress in Brain Research, volume 155, 2006.

[12] L. W. Renninger and J. Malik. When is scene recognition just texture recognition? Vision Research, 44:2301-2311, 2004.

[13] I. Simon, N. Snavely, and S. M. Seitz. Scene summarization for online image collections. In Proc. ICCV, 2007.

[14] N. Snavely, S. M. Seitz, and R. Szeliski. Photo tourism: exploring photo collections in 3d. SIGGRAPH, 25(3), 2006.

[15] R. Szeliski. "Where am I?": ICCV 2005 Computer Vision Contest. http://research.microsoft.com/iccv2005/Contest/.

[16] W. Thompson, C. Valiquette, B. Bennett, and K. Sutherland. Geometric reasoning for map-based localization. Spatial Cognition and Computation, 1(3), 1999.

[17] A. Torralba, R. Fergus, and W. T. Freeman. Tiny images. Technical Report MIT-CSAIL-TR-2007-024, 2007.

[18] J. Vogel and B. Schiele. Semantic modeling of natural scenes for content-based image retrieval. Int. J. Comput. Vision, 72(2):133-157, 2007.

[19] W. Zhang and J. Kosecka. Image based localization in urban environments. In 3DPVT '06, 2006. 


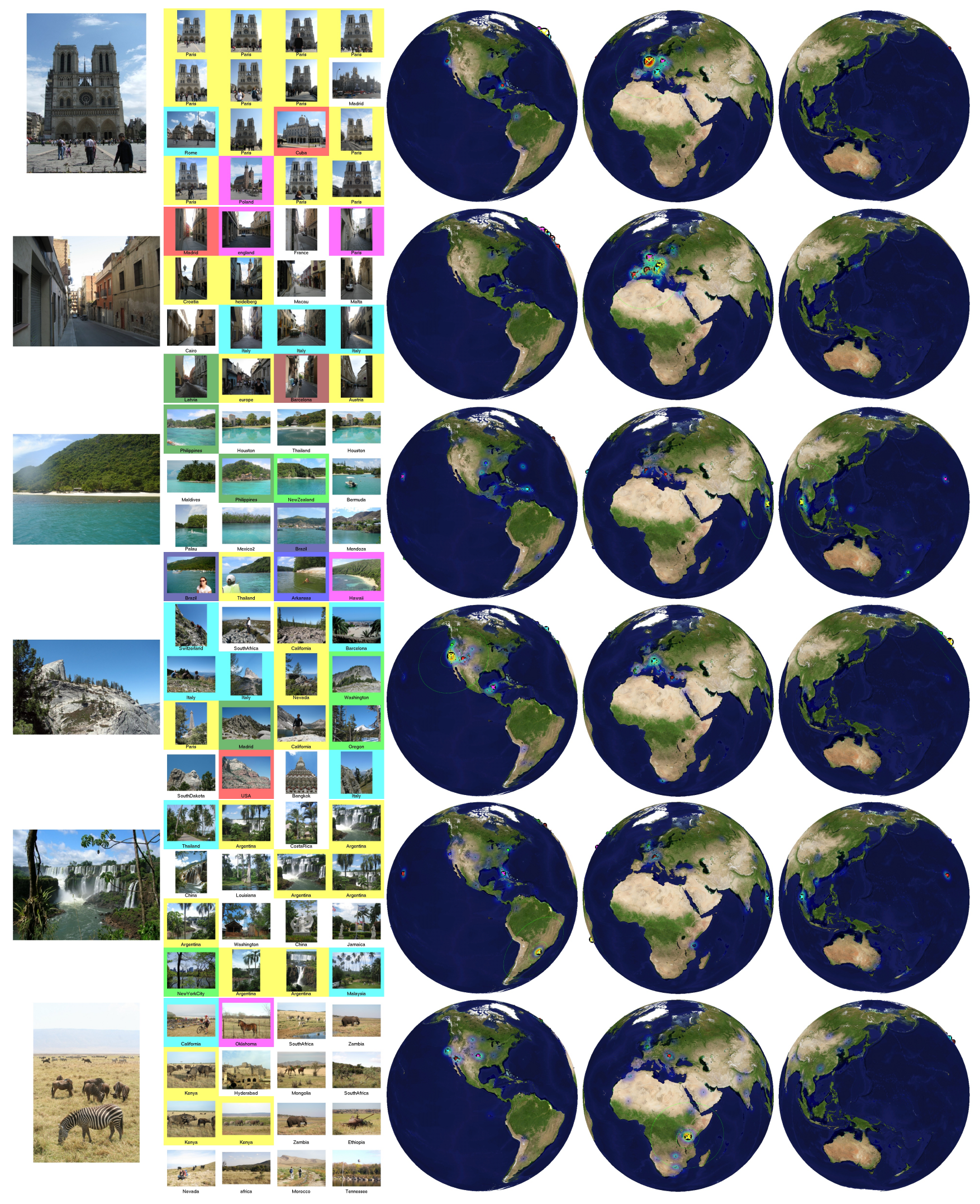

Figure 15. From left to right: query images, nearest neighbors, and three visualizations of the estimated geolocation probability map. The probability map is shown as a jet-colorspace overlay on the world map. Cluster modes are marked with circumscribed "X"'s whose sizes are proportional to cluster cardinality. If a scene match is contained in a cluster it is highlighted with the corresponding color. The ground truth location is a cyan asterisk surrounding by green contours at radii of $200 \mathrm{~km}, 750 \mathrm{~km}$, and $2500 \mathrm{~km}$. From top to bottom, these photos were taken in Paris, Barcelona, Thailand, California, Argentina, and Tanzania. For the Yosemite, California query note that the apparently spurious "Paris" match with the Eiffel tower is in fact the Paris Casino in nearby Las Vegas. Perhaps the texture similarities from vegetation and color distribution similarities helped produce this informative match. 